\title{
APRESENTACCÃO DA TÉCNICA DE ESTUdO DO TEMPO DE ESVAZIAMENTO GÁSTRICO POR MEIO DA ULTRA-SONOGRAFIA*
}

\author{
Cristina Pirani Valadares ${ }^{1}$, Rogério Augusto Pinto Silva ${ }^{2}$, Wilson Campos Tavares Junior ${ }^{3}$, \\ Marco Antonio Duarte ${ }^{4}$
}

Resumo OBJETIVO: Descrever a técnica de avaliação do esvaziamento gástrico em crianças. MATERIAIS E MÉTODOS: Foram estudadas 14 crianças voluntárias saudáveis, com idades de 2 a 11 anos. As crianças ingeriram leite modificado, na proporção de $200 \mathrm{ml} / \mathrm{m}^{2}$ de superfície corporal. A área do antro gástrico foi medida em todos os pacientes no tempo zero, antes da ingestão do meio de contraste, e aos 60, 90, 120 e 150 minutos após a ingestão do meio de contraste. RESULTADOS: A dieta foi bem tolerada pelos pacientes. Foi observado que em 150 minutos após a ingestão do alimento, 85\% dos pacientes apresentaram-se com esvaziamento gástrico total. CONCLUSÃO: A ultra-sonografia é método seguro e barato, sendo uma alternativa para o estudo do esvaziamento gástrico.

Unitermos: Ultra-sonografia; Motilidade gástrica; Esvaziamento gástrico.

Abstract Technique for the evaluation of gastric emptying time using ultrasound.

OBJECTIVE: To describe a technique for measuring gastric emptying in children. MATERIALS AND METHODS: Gastric emptying time was measured in 14 healthy volunteer children aged between 2 to 11 . The children were asked to drink modified milk in proportion of $200 \mathrm{ml} / \mathrm{m}^{2}$ of body surface. The antral area was measured before the injection of contrast media and at 60, 90, 120 and 150 minutes after the injection. RESULTS: The diet was well tolerated by most patients. In the majority $(85 \%)$ of the children total gastric emptying occurred 150 minutes after the ingestion of the solution. CONCLUSION: Ultrasound is a safe and low cost alternative for the assessment of gastric emptying.

Keywords: Ultrasound; Gastric motility; Gastric emptying.

\section{INTRODUÇÃO}

O estudo ultra-sonográfico do esvaziamento gástrico é técnica ainda pouco utilizada em nosso meio, sendo a cintilografia considerada, atualmente, o padrão-ouro para a avaliação do trânsito gástrico. O estudo do esvaziamento gástrico se presta a identificar, caracterizar e estabelecer possíveis correlações fisiopatológicas, sendo também efetiva para testar a eficiência de uma terapêutica medicamentosa ou cirúr-

* Trabalho realizado pelo Grupo de Gastroenterologia Pediátrica e Departamento de Radiologia e Diagnóstico por Imagem do Hospital das Clínicas da Universidade Federal de Minas Gerais (HC-UFMG) e Centro Especializado em Ultra-som (CEU), Belo Horizonte, MG.

1. Médica Gastroenterologista Pediátrica, Assistente do Serviço de Gastroenterologia Pediátrica do HC-UFMG.

2. Médico Ultra-sonografista do Setor de Ultra-sonografia do Departamento de Radiologia e Diagnóstico por Imagem do HCUFMG.

3. Médico Residente de Radiologia e Diagnóstico por Imagem do HC-UFMG.

4. Professor Adjunto, Doutor do Departamento de Pediatria, Serviço de Gastroenterologia Pediátrica da Faculdade de Medicina da UFMG.

Endereço para correspondência: Dr. Wilson Campos Tavares Junior. Rua Alagoas, 581, ap. 1704, Funcionários. Belo Horizonte, MG, 30130-160. E-mail: wilsontavaresjrmd@yahoo.fr

Recebido para publicação em 12/2/2005. Aceito, após revisão, em 18/5/2005. gica $^{(1-3)}$. O estudo do esvaziamento tem permitido a identificação tanto de trânsito rápido como lento em pacientes previamente diagnosticados como tendo base psicológica para suas queixas digestivas ${ }^{(\mathbf{1})}$.

As diversas técnicas que avaliam o esvaziamento gástrico visam a definir, a partir de um conteúdo gástrico inicial de valor conhecido, a taxa de eliminação desse conteúdo pelo estômago. Dentre essas técnicas, incluem-se as de intubação-aspiração, a radiológica, a cintilográfica, a ressonância magnética, a ultra-sonografia, a pletismografia de impedância, a tomografia de impedância, os testes respiratórios, os de absorção de fármacos e o teste do traçador magnético ${ }^{(2,4,5)}$.

Apesar da diversidade de técnicas propostas e utilizadas no estudo do esvaziamento gástrico, ainda há muito que se esclarecer na fisiologia desse processo ${ }^{(6-8)}$. As dificuldades de execução e o pouco apuro na interpretação dos resultados são os fatores apontados como principais responsáveis pela limitação das técnicas ${ }^{(\mathbf{9 , 1 0})}$.

Um bom método para a avaliação da motilidade e do esvaziamento gástrico deve ser de fácil acesso, reprodutível, baixo custo, de preferência não-invasivo ${ }^{(11)}$. Deve ser capaz de permitir a visualização da motilidade do antro, do piloro e do duodeno, bem como do fluxo transpilórico. Deve permitir, também, a caracterização da frequiência e da amplitude das contrações antrais e ser capaz de tornar possível a análise da correlação entre a motilidade e o esvaziamento gástrico ${ }^{(12)}$. A ultra-sonografia oferece estas possibilidades, sendo um método dinâmico que permite a visualização em tempo real da motilidade e do esvaziamento gástrico.

\section{MATERIAIS E MÉTODOS}

Avaliou-se o esvaziamento gástrico de 14 voluntários sadios - sete do sexo feminino e sete do sexo masculino - com idades compreendidas entre 2 e 11 anos e média de 8 anos. Os 14 voluntários foram selecionados de um grupo acompanhado em ambulatório periférico, que excluiu indivíduos que apresentavam sintomas relacionados ao tubo digestivo ou doenças que poderiam interferir nos resultados. 
Todos os indivíduos selecionados foram informados do teor da pesquisa e assinaram termo de consentimento, sendo este estudo aprovado no Comitê de Ética da Instituição onde o trabalho foi realizado, sob o protocolo COEP 405/04.

Os exames ultra-sonográficos foram realizados em aparelho modelo SSD-125 (Toshiba, Tóquio, Japão), utilizando-se transdutor linear com frequiência central de 7,5 $\mathrm{MHz}$ e sonda convexa de 3,75 $\mathrm{MHz}$. As crianças foram investigadas em jejum, após a ingestão da dieta padrão (tempo zero) e aos 60, 90, 120 e 150 minutos subsequientes. Os exames foram realizados em ortostatismo.

As medidas do antro gástrico foram realizadas utilizando-se a parede externa do estômago.

Para o estudo do esvaziamento gástrico, foi avaliada a área da secção transversa utilizando-se técnica desenvolvida por Bolondi et al. ${ }^{(\mathbf{1 3 - 1 6 )}}$, através de corte longitudinal na região epigástrica, usando-se por referência o plano sagital que passa pela aorta e veia mesentérica superior, através da fórmula da área da elipse dada por:

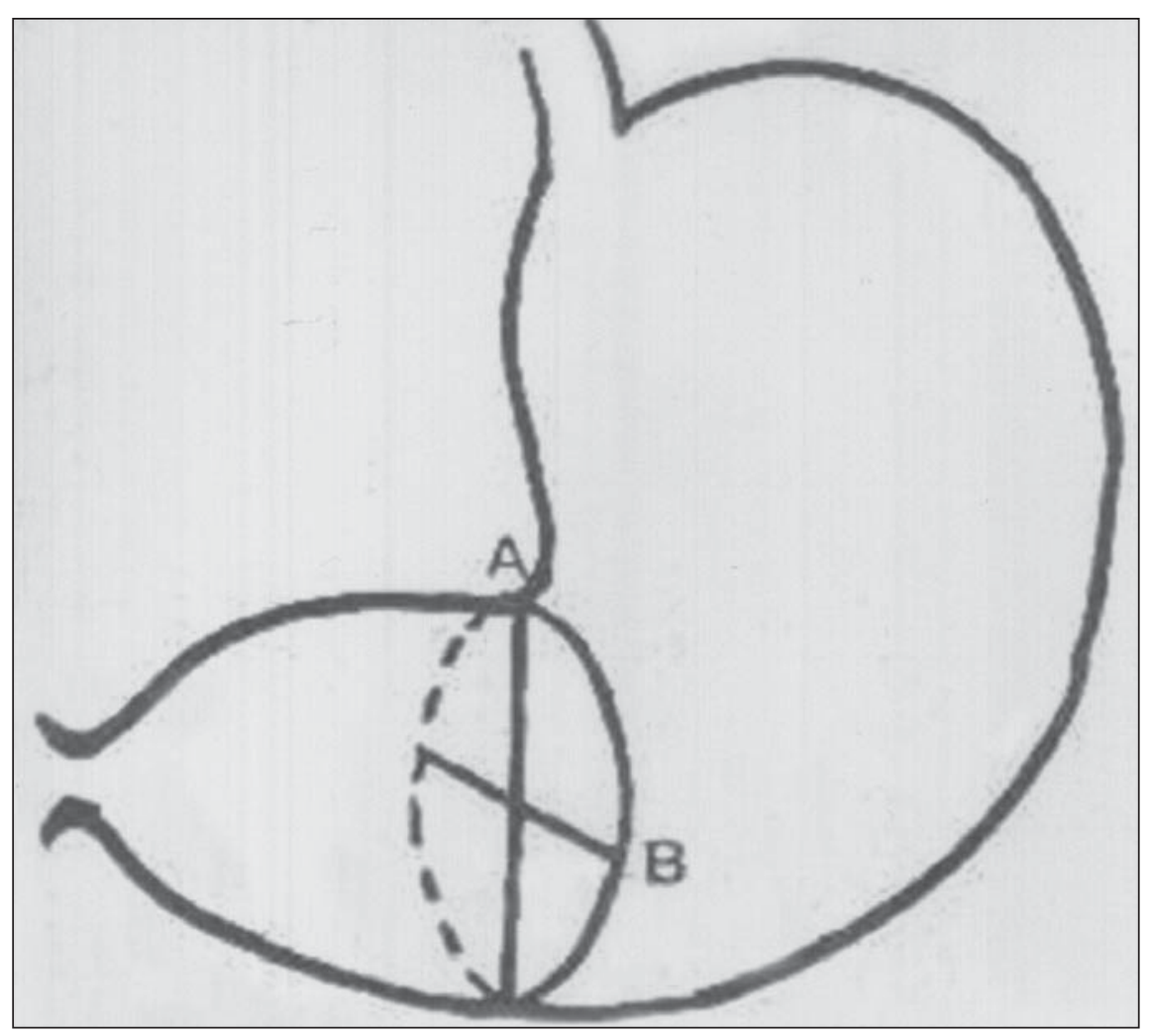

Figura 1. Apresenta os diâmetros longitudinais e ântero-posteriores que devem ser avaliados para se definir a área de secção.
Não se observaram diferenças significativas quanto ao tempo de esvaziamento gástrico e ao comportamento dinâmico do estômago que pudessem ser correlacionadas com sexo e idade dos voluntários. Em todas as crianças pudemos observar, após o início do efetivo esvaziamento, que o conteúdo gástrico prosseguiu sendo eliminado para o delgado de forma gradual e contínua. Aos 150 minutos, 86\% dos pacientes apresentaram esvaziamento completo do estômago, visualizado na ultrasonografia (Figura 2).

\section{DISCUSSÃO}

O uso de uma refeição líquida com lipídios, carboidratos e proteínas, que pode ser reprodutível e em proporções semelhantes para o perfil biofísico de cada indivíduo, possibilitou a avaliação da motilidade e do esvaziamento do estômago de maneira uniforme. Além disso, por já ser líquida, esta dieta diminui a interferência do gás, diminuindo assim os erros por dificuldades de leitura, configurando-se em mistura adequada para aferição do esvaziamento gástrico.

O relaxamento receptivo, a contratilidade coordenada que integra antro, piloro e duodeno, bem como o comportamento peristáltico do período pós-prandial, podem efetivamente ser observados ${ }^{(17,18)}$.

As imagens ultra-sonográficas permitem analisar a repleção e a sequiência dinâmica do esvaziamento gástrico. Permitem identificar o ponto de início da contração, a migração da onda de contração pelo corpo e antro, a abertura pilórica e o fluxo transpilórico ${ }^{(\mathbf{1 2})}$. Este é um método de fácil metodização, baixo custo e boa reprodutibilidade ${ }^{(19)}$.

Mesmo com a utilização de diversos métodos para a avaliação do esvaziamento gástrico $^{(20)}$, ainda há muito a esclarecer em sua fisiologia ${ }^{(21)}$, sendo vantajosa a visão direta e em tempo real permitida pela ultra-sonografia.

No presente estudo, o tempo de $150 \mathrm{mi}$ nutos foi utilizado como ponto de corte, pois foi observado esvaziamento gástrico em $86 \%$ dos voluntários estudados.

A pesquisa realizada autoriza-nos a concluir que a ultra-sonografia permite, com adequada resolução, a análise quali- 


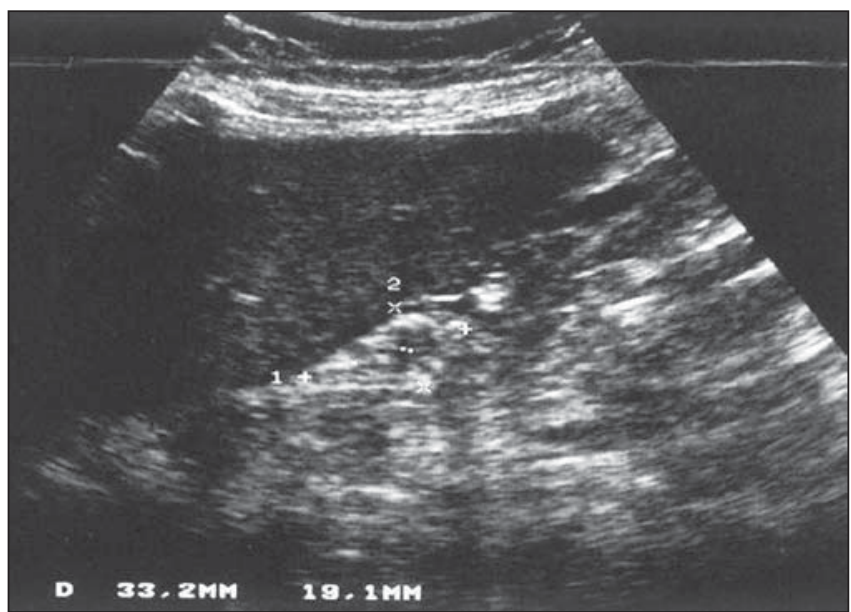

A

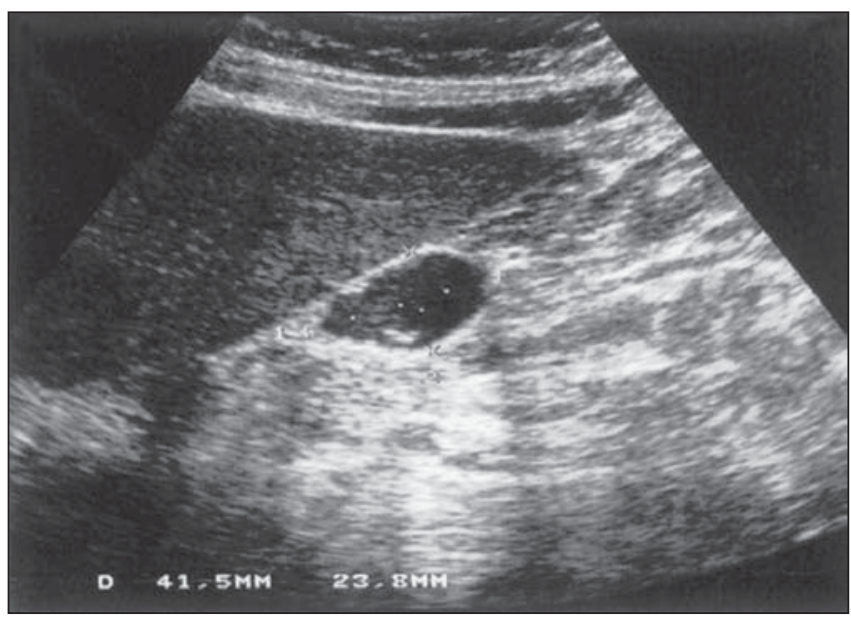

C

Figura 2. Estudo em paciente do sexo masculino, de nove anos de idade, mostra as medidas do antro gástrico com o paciente em jejum (A) e aos 60 minutos (B), 90 minutos (C), 120 minutos (D) e 150 minutos (E) após ingestão do alimento, em que se observou que a secção gástrica retornou a valores de jejum.

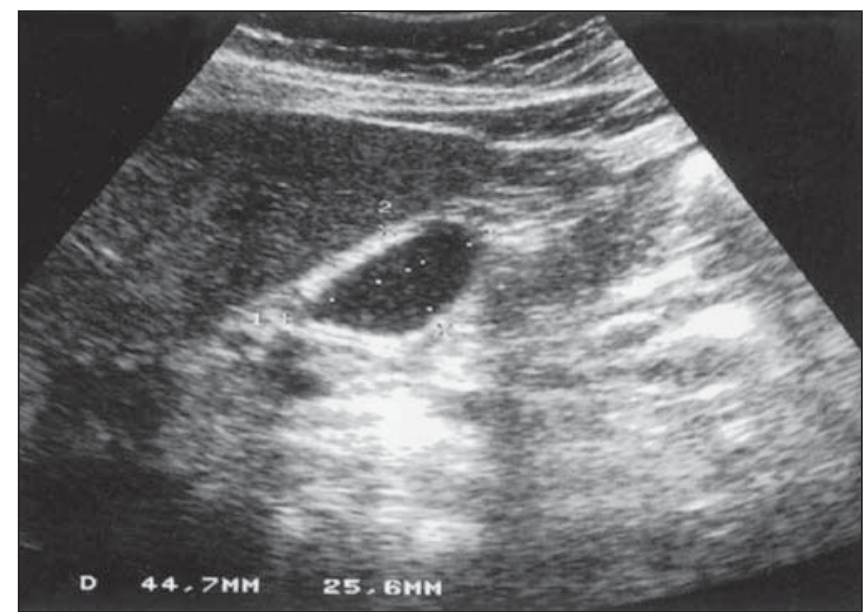

B

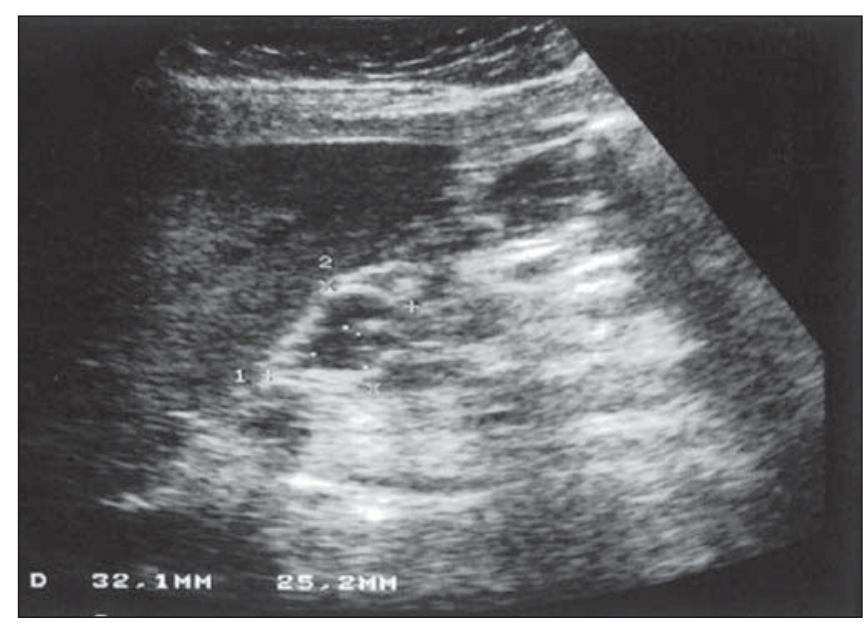

D

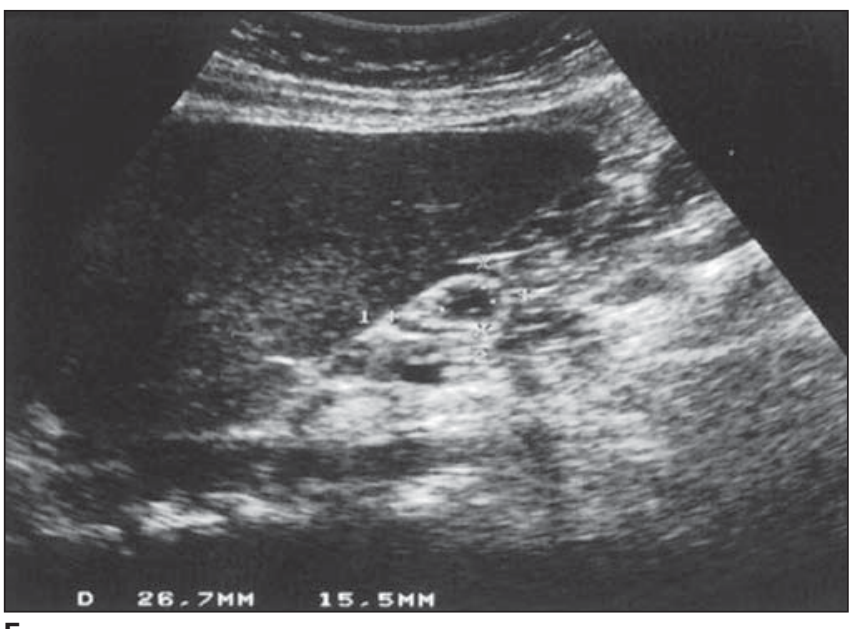

E

\section{REFERÊNCIAS}

tativa da motilidade e do esvaziamento gástrico. Os nossos resultados expressam um índice de esvaziamento gástrico, determinado pela variação da área de projeção gástrica, em imagens radiológicas obtidas em incidência póstero-anterior.
1. Malmud LS, Vitti RA. Gastric emptying. J Nucl Med 1990;31:1499-1500.

2. Rezende-Filho J. Esvaziamento gástrico: métodos de avaliação. In: Castro LP, Savassi-Rocha PR, Cunha-Melo JR. Tópicos em gastroenterologia 5. Rio de Janeiro: Medsi, 1994;35-47.
3. Chang TM, Passaro EP Jr, Su DJ, et al. Technetium 99m-DTPA microcapsules: a new preparation for gastric emptying studies. Am J Surg 1986;151: 722-724.

4. Horowitz M, Dent J, Fraser R, Sun W, Hebbard G. Role and integration of mechanisms controlling gastric emptying. Dig Dis Sci 1994;39(12 Suppl): 7S-13S. 
5. Rezende-Filho J. Métodos de avaliação da motilidade gástrica. São Paulo: Resumos do II Simpósio de Motilidade Digestiva, 1996;11-14.

6. Berstad A, Hausken T, Gilja OH, Thune N, Matre $\mathrm{K}$, Odegaard S. Volume measurements of gastric antrum by 3-D ultrasonography and flow measurements through the pylorus by duplex technique. Dig Dis Sci 1994;39(12 Suppl):97S-100S.

7. Parkman HP, Harris AD, Krevsky B, Urbain JL, Maurer AH, Fisher RS. Gastroduodenal motility and dysmotility: an update on techniques available for evaluation. Am J Gastroenterol 1995;90:869892.

8. Urbain JL, Charkes ND. Recent advances in gastric emptying scintigraphy. Semin Nucl Med 1995;25: 318-325.

9. Vantrappen G. Methods to study gastric emptying. Dig Dis Sci 1994;39(12 Suppl):91S-94S.

10. Benini L, Sembenini C, Heading RC, et al. Simultaneous measurement of gastric emptying of a solid meal by ultrasound and by scintigraphy. Am J Gastroenterol 1999;94:2861-2865.
11. Gomes H, Hornoy P, Liehn JC. Ultrasonography and gastric emptying in children: validation of a sonographic method and determination of physiological and pathological patterns. Pediatr Radiol 2003;33: 522-529.

12. Gilja OD, Heimdal A, Hausken T, et al. Strain during gastric contractions can be measured using Doppler ultrasonography. Ultrasound Med Biol 2002;28:1457-1465.

13. Bolondi L, Bortolotti M, Santi V, Calletti T, Gaiani S, Labò G. Measurement of gastric emptying time by real-time ultrasonography. Gastroenterology 1985;89:752-759.

14. Cucchiara S, Minella R, Iorio R, et al. Real-time ultrasound reveals gastric motor abnormalities in children investigated for dyspeptic symptoms. J Pediatr Gastroenterol Nutr 1995;21:446-453.

15. Ricci R, Bontempo I, Corazziari E, La Bella A, Torsoli A. Real time ultrasonography of gastric antrum. Gut 1993;34:173-176.

16. Dumitrascu DL, Barnert J, Kirschner T, Wienbeck M. Antral emptying of semisolid meal measured by real-time ultrasonography in chronic renal failure. Dig Dis Sci 1995;40:636-644.

17. McCallum RW. Motor function of the stomach in health and disease. In: Sleisenger MH, Fordtran JS, editors. Gastrointestinal disease: pathophysiology, diagnosis, management. 4th ed. Philadelphia: WB Saunders, 1989;675-713.

18. Kusunoki H, Haruma K, Hata J, et al. Real-time ultrasonographic assessment of antroduodenal motility after ingestion of solid and liquid meals by patients with functional dyspepsia. J Gastroenterol Hepatol 2000;15:1022-1027.

19. Pedersen JF. A modified sonographic technique for assessment of gastric emptying of liquid. Acta Radiol 2003;44:340-342.

20. Livoti G, Tulone V, Bruno R, et al. Ultrasonography and gastric emptying: evaluation in infants with gastroesophageal reflux. J Pediatr Gastroenterol Nutr 1992;14:397-399.

21. Malagelada JR. Conscious perception of gut activity. Dig Dis Sci 1994;39(12 Suppl):51S-53S 